\title{
Real Time Simulation of Power Electronic Converters
}

\author{
Babu Naik G ${ }^{1}$, Shilpa G' ${ }^{2}$, H.D. Kattimani ${ }^{3}$, Ozwin Dominic Dsouza ${ }^{4}$ \\ Assistant Professor, EEE Dept. BMS Institute of Technology \& Management, \\ Yelahanka, Bangalore, Karnataka, India ${ }^{1,2,4}$ \\ Associate Professor, EEE Dept. BMS Institute of Technology \& Management, \\ Yelahanka, Bangalore, Karnataka, India ${ }^{3}$
}

\begin{abstract}
In this paper, designing a real time simulator and models of boost converter are solved. By using real time simulator it is possible to simulate power converter circuits accurately. They can be efficiently used in various fields such as industries, education, etc. With this, simulator can be used for performance optimization and safety engineering in various fields. Building a real time simulator Results obtained from real time simulation are compared with offline simulation. The models are solved by using MAT Lab and simulator. Also various other activities such as solving differential equations, performing basic operations on signals, realizing low pass filter is also done by using both simulation processes.
\end{abstract}

Keywords: Simulator, Mat Lab and MP Lab.

\section{INTRODUCTION}

Simulation is an act or process of imitating the real world operations or systems. Simulation is usually performed to ensure safety of hardware before manufacturing any device or system and performance optimization. In order to perform real time simulation, a simulator is needed to be designed. With the development of rail propulsion transportation including high speed electrical train, urban subway and Maglev, high power converter and control system play significant roles to deal with high speed and safe operation. However, control systems for high power converter fed rail traction transportation are awfully more complex than that for previous vehicles [1]. So, its functions test is very important before on-site installation, commissioning. When a single large time step is used for the whole network, the simulator can capture switching events only at sampling instants so that multiple switching that may occur within a time step are missed [2].

A more important approach is to interface the actual digital controller and protection devices with a real-time simulator, their performance under actual operating conditions can be evaluated thoroughly to check whether the performance reaches the deign standard or not. This way is also known as hardware-in-the-loop (HIL) simulation, which replaces the emulated hardware under test or control logic in the simulation model with real hardware that interacts with the computer models The chip DSPIC30F4011 is used[3]. DSC chip can be coded by using assembly or $\mathrm{C}$ language. Overall simulator is a device which can take input and process it and give respective output that represents behaviour of the system. To perform real time simulation, understanding the behaviour of the system is the first step [4]. The behaviour can be implemented digitally by deriving mathematical model of the system and coding it into chip. Power converter circuits such as buck, boost converter circuits are used in manipulating dc voltage levels. Simulating such circuits include solving differential equations, performing basic operations on electric signals etc. In this paper simulation of boost converter by using ideal and exact model is done.

\section{SOFTWARE REQUIREMENTS}

\section{A. Matlab}

MATLAB is a high-level language and interactive environment for numerical computation, visualization, and programming. Using MATLAB, you can analyze data, develop algorithms, and create models and applications. The language, tools, and built-in math functions enable you to explore multiple approaches and reach a solution faster than with spreadsheets or traditional programming languages, such as $\mathrm{C} / \mathrm{C}++$ or Java. You can use MATLAB for a range of applications, including signal processing and communications, image and video processing, control systems, test and measurement, computational finance, and computational biology. More than a million engineers and scientists in industry and academia use MATLAB, the language of technical computing.

- High-level language for numerical computation, visualization, and application development

- Interactive environment for iterative exploration, design, and problem solving

- Mathematical functions for linear algebra, statistics, Fourier analysis, filtering, optimization, numerical integration, and solving ordinary differential equations

- Built-in graphics for visualizing data and tools for creating custom plots

- Development tools for improving code quality and maintainability and maximizing performance

- Tools for building applications with custom graphical interfaces 
- Functions for integrating MATLAB based algorithms with external applications and languages such as $\mathrm{C}$, Java, .NET, and Microsoft Excel

\section{B. MP LAB}

MPLAB is a free integrated development environment for the development of embedded applications on PIC and dsPICchip and is developed by Microchip technology. or It is an executable program that integrates a compiler, an assembler, a project manager, an editor, a debugger, simulator, and an assortment of other tools within one Windows application. MPLAB IDE runs as a 32-bit applications on MS windows is easy to use and includes a host of free software components for fast applications development and super-charged debugging. MPLAB IDE also serves as a single, unified graphical user interface for additional microchip and third party software and hardware development tools. Moving between tools is a snap and upgrading from the free software simulator to hardware debug and programming tools is done in a flash because MPLAB IDE has the same user interface for all tools. simulator runs from a HEX file that can be programmed into the dsPIC. For the simulator to run we must first create a source code file and successfully assemble the source code.

- Flexible customizable programmer's text editor.

- Fully integrated debugging with right mouse click menus for breakpoints, trace and editor functions.

- Tabbed editor option or separate source windows.

- Recordable macros

- Context sensitive colour highlighting for assembly, c and basic code readability.

- Mouse over variable to instantly the contents of variables and registers.

- Set breakpoints and trace points directly in editor to instantly make changes and evaluate their effects.

- Graphical project manager.

- Version control for MS Source Safe, CVS, PVCS, Subversion.

\section{HARDWARE REQUIREMENTS}

\section{A. DSPIC30F4011 CHIP}

Architecture of dsPIC30F4011 chip. The dsPIC microcontroller is a part of Microchip's 16-Bit microcontroller family. The architecture of dsPIC or Digital Signal Controller is same as the architecture withPIC16bitfamily, with additional blocks of Digital Signal Processing. It consists of $40 \mathrm{I} / \mathrm{O}$ pins for communication to various parts in a kit. It uses Harvard architecture with an enhanced instruction set. The CPU has a 24-bit instruction word, with a variable length op code field. The program counter (PC) is 24-bits wide and addresses up to $4 \mathrm{M} \times 24$ bits of user program memory space. A single cycle instruction pre-fetch mechanism is used to help maintain throughput and provides predictable execution.

The dsPIC30F devices have sixteen 16-bit working registers in programmer's model. Each of the working registers can act as a data, address, or address offset register.

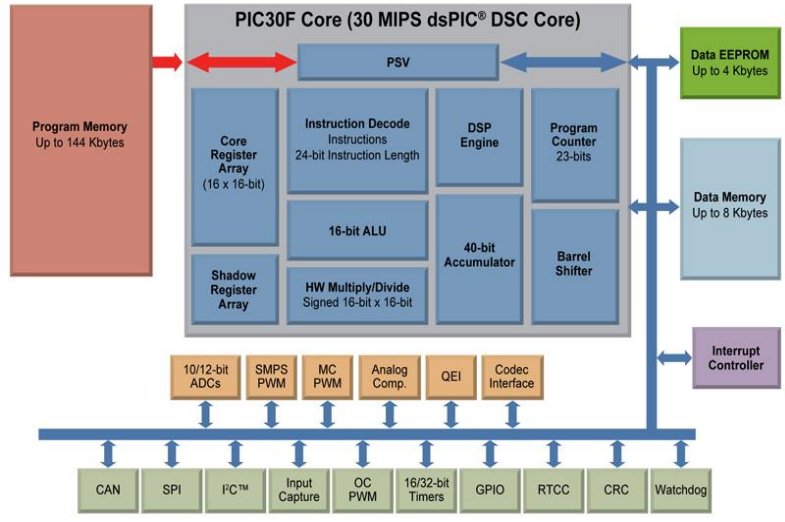

Fig 1: DSPIC30F4011 CHIP

The 16th working register (W15) operates as a software stack pointer for interrupts and calls. The dsPIC30F instruction set has two classes of instructions: the MCU class of instructions and the DSP class of instructions. These two instruction classes are seamlessly integrated into the architecture and execute from a single execution unit. For most instructions, the dsPIC 30F is capable of executing a data (or program data) memory read, a working register (data) read, a data memory write and a program (instruction) memory read per instruction cycle.

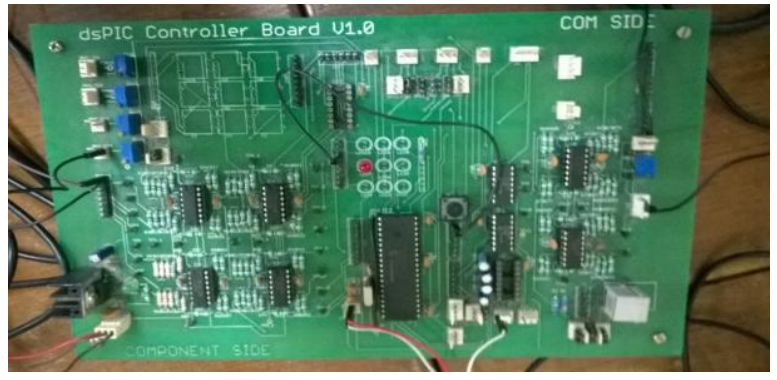

Fig 2: Trainer Kit

\section{B. Signal Conditioning Circuits}

This circuit does the function of reducing the Input voltage level to the chip. The $230 \mathrm{~V}$ input voltage will be reduced to $+/-15 \mathrm{~V}$ by step down transformer. After all losses in resistor and capacitor circuits the voltage available will be +/- 10V. In the circuit 4 opamps are present in which 2 opamps are useful. The first opamp divides $10 \mathrm{~V}$ by 4 to get $+/-2.5 \mathrm{~V}$ and the second opamp shifts the voltage level to $+5 \mathrm{~V}$, this voltage level will be input to the chip. The chip with ADC converts analog signal to digital signal. 2 DAC are used which does the reverse process of signal conditioner circuit. Finally analog signal output will be given into signal generator in which waveforms are shown.

The IC used in DAC is MCP4922.

The IC used in ADC is TL084P.

The IC used in power side is IC7805.

The IC used in COM is MAX232.

C. MCP4922

The MCP4922 device is a dual channel 12-bit DAC which uses an external voltage reference. This device offers high accuracy and low power consumption, and is available in 
various packages. Communication with the device is accomplished via a simple serial interface using SPI protocols. The MCP4922 device is a part of the MCP4902/MCP4912/MCP4922 product family, which are dual channel 8-bit/10-bit/12-bit DACs which use external voltage reference (VREF). These devices provide very high accuracy and low noise performance, and are suitable for consumer and industrial applications, such as set point control, offset adjustment and sensor calibration applications. The low power consumption and small package options make these devices very attractive for many portable and battery-powered applications. If only a single output is needed then the MCP4901/4911/4921 single channel product family can be used.

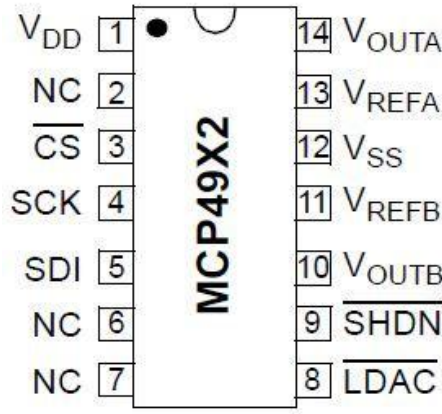

Fig 3: Pin Diagram MCP49X2

- 12-bit Resolution

- Dual Channel Voltage Output

- $2.7 \mathrm{~V}$ to $5.5 \mathrm{~V}$ Operation

- Operating Current $350 \mu \mathrm{A}$ (typ)

- External Voltage Reference Pin

- INL \pm 2 LSB (typ)

- $\mathrm{DNL} \pm 0.75 \mathrm{LSB}(\max )$

- Output Settling Time $4.5 \mu \mathrm{s}$

- SPI Interface

- 14-pin PDIP, SOIC, and TSSOP packages

- Temperature Range $-40^{\circ} \mathrm{C}$ to $+125^{\circ}$

D. ICTL084P Description

The TL08xx JFET-input operational amplifier family is designed to offer a wider selection than any previously developed operational amplifier family. Each of these JFET-input operational amplifiers incorporates wellmatched, high-voltage JFET and bipolar transistors in a monolithic integrated circuit. The devices feature high slew rates, low input bias and offset currents, and low offset-voltage temperature coefficient.

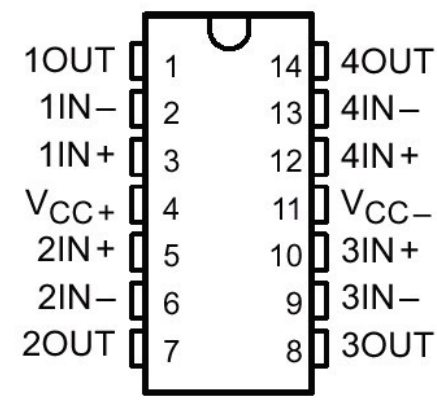

Fig 4: Pin Diagram
- Low Power Consumption: $1.4 \mathrm{~mA} / \mathrm{ch}$ Typical

- Wide Common-Mode and Differential Voltage Ranges

- Low Input Bias Current: 30 pA Typical

- Low Input Offset Current: 5 pA Typical

- Output Short-Circuit Protection

- Low Total Harmonic Distortion: 0.003\% Typical

- High Input Impedance: JFET Input Stage

- Latch-Up-Free Operation

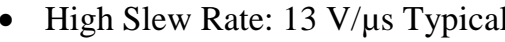

- Common-Mode Input Voltage Range

E. 5 IC 7805

7805 is a voltage regulator integrated circuit. It is a member of $78 \mathrm{xx}$ series of fixed linear voltage regulator ICs. The voltage source in a circuit may have fluctuations and would not give the fixed voltage output. The voltage regulator IC maintains the output voltage at a constant value. The $\mathrm{xx}$ in $78 \mathrm{xx}$ indicates the fixed output voltage it is designed to provide. 7805 provides $+5 \mathrm{~V}$ regulated power supply. Capacitors of suitable values can be connected at input and output pins depending upon the respective voltage levels.

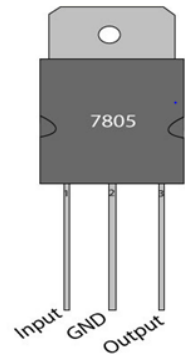

Fig5: Pin Diagram

\begin{tabular}{|c|c|c|}
\hline Pin No & Function & Name \\
\hline 1 & Input voltage (5V-18V) & Input \\
\hline 2 & Ground (0V) & Ground \\
\hline 3 & $\begin{array}{c}\text { Regulated output; 5V } \\
(4.8 \mathrm{~V}-5.2 \mathrm{~V})\end{array}$ & Output \\
\hline
\end{tabular}

Table 1: Pin Description

F. FT232RL

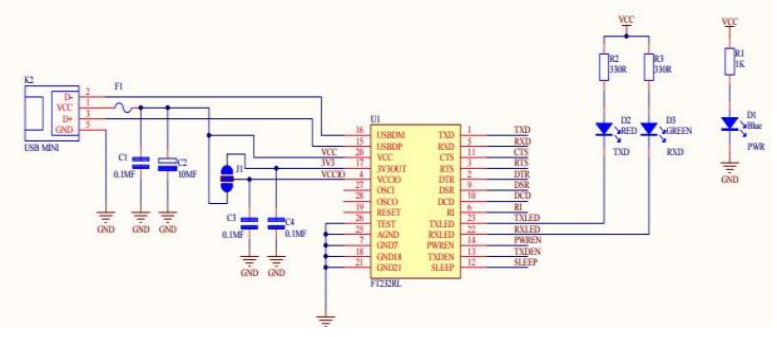

Fig 6: FT232RL

The FT232R is the latest device to be added to FTDI's range of USB UART interface Integrated Circuit Devices. The FT232R is a USB to serial UART interface with optional clock generator output, and the new FTDI ChipID $^{\text {TM }}$ security dongle feature. In addition, asynchronous and synchronous bit bang interface modes are available. USB to serial designs using the FT232R have been further 
simplified by fully integrating the external EEPROM, The simulator consists of DSC chip, Signal conditioner clock circuit and USB resistors onto the device. The circuit, ADC, DAC, Power supply, USB Interface, and FT232R adds two new functions compared with its output CRO. The Input of voltage $+15 \mathrm{~V}$ to $-15 \mathrm{~V}$ is given predecessors, effectively making it a "3-in-1" chip for to signal conditioner circuit after losses $10 \mathrm{~V}$ will be some application areas. The internally generated clock available. This voltage after conditioning and level $(6 \mathrm{MHz}, 12 \mathrm{MHz}, 24 \mathrm{MHz}$, and $48 \mathrm{MHz})$ can be brought out shifting voltage of $5 \mathrm{~V}$ is available to ADC in which analog of the device and used to drive a microcontroller or signal is converted into Digital signal. The programmed external logic. A unique number (the FTDI Chip-ID ${ }^{\mathrm{TM}}$ ) is will be burned into simulator after simulation the output burnt into the device during manufacture and is readable over USB, thus forming the basis of a security dongle which can be used to protect customer application software from being copied.

- Cellular and Cordless Phone USB data transfer cables and interfaces

- Interfacing MCU/PLD/FPGA based designs to USB

- USB Audio and Low Bandwidth Video data transfer

- PDA to USB data transfer

- USB Smart Card Readers

- USB Instrumentation

\section{SCOPE OF PRESENT WORK}

The project aims on designing a real time simulator and simulate boost converter by solving ideal and exact mathematic models

A. Specific objectives

- Designing a simulator using DSPIC30F4011 chip.

- Performing basic operations on signals using simulator and MAT Lab.

- Solving differential equations using simulator and MAT Lab.

- Deriving ideal and exact mathematical model of boost converter and low pass filter.

- Solving mathematical models using simulator and MAT Lab and PSIM.

- Comparing the results.

In this project the simulation of converters are done through offline and online, In offline the mathematical model of the converters are programmed in MATLAB and the output is taken. And other part is to build the simulator in which the converters are programmed in MPLAB and the programmes are burned into the simulator, the output is seen on the signal generator. The output result of both simulations is compared.

\section{B. Block Diagram of Simulator}

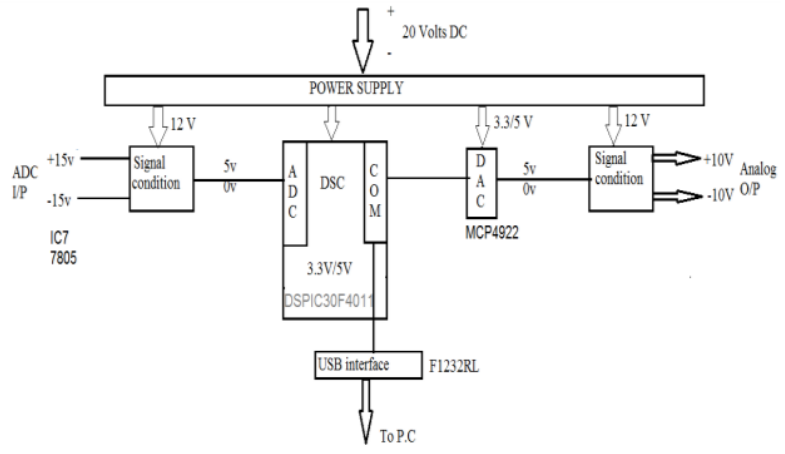

Fig 7: Block Diagram of Stimulator

Output.

C. Basic operations on signals

Signals: A signal is a function representing a physical quantity or variable, and typically it contains information about the behavior or nature of the phenomenon.

Continuous-time: Signals are analog or digital signals such that their magnitudes are defined for all values of $t$. A continuous-time signal is an infinite and uncountable set of numbers, as are the possible values each number can have. That is, between a start and end time, there are infinite possible values for time $t$ and instantaneous amplitude, $x(t)$. And the variable of time is continuous.

Discrete-time: Signals are analog or digital signals with magnitudes that are defined at specific instants of time only and are undefined for other time instants. In a discrete-time signal, the number of elements in the set, as well as the possible values of each element, is finite, countable, and can be represented with computer bits, and stored on a digital storage medium. And the variable of time is discrete.

Addition: Physical significance of this operation is to add two signals like in the addition of the background music along with the human audio. Another example is the undesired addition of noise along with the desired audio signals.

$$
y(t)=x 1(t)+x 2(t)
$$

Multiplication: Convolution in the time domain corresponds to multiplication in the frequency domain. It turns out that this relationship is symmetric, in that multiplication in the time domain corresponds to a peculiar form of convolution in the frequency domain.

$$
y(t)=x 1(t) \cdot x 2(t)
$$

\section{DIFFRENTIAL EQUATIONS}

If $\mathrm{y}=\mathrm{f}(\mathrm{x})$ is an unknown function, an equation which involves at least one derivative of $\mathrm{y}$ with respect to $\mathrm{x}$ is called an differential equation.

Finding $y$ as a function of $x$ explicitly $[\mathrm{y}=\mathrm{f}(\mathrm{x})$ ] or a relationship in $x$ and $y$ satisfying the D.E $[f(x, y)=c$ ] constitutes the solution of the D.E. In applications, the functions usually represent physical quantities, the derivatives represent their rates of change, and the equation defines a relationship between the two. Because such relations are extremely common, differential equations play a prominent role in many disciplines including engineering, physics, economics, and biology. In pure mathematics, differential equations are studied from several different perspectives, mostly concerned with their solutions - the set of functions that satisfy the 
equation. Only the simplest differential equations are the following modified euler's formula, where the solvable by explicit formulas; however, some properties of successive approximations are denoted by y1(1), y1(2), solutions of a given differential equation may be $y 1(3), \ldots$ etc. determined without finding their exact form. If a selfcontained formula for the solution is not available, the solution may be numerically approximated using computers. The theory of dynamic systems puts emphasis on qualitative analysis of systems described by differential equations, while many numerical methods have been developed to determine solutions with a given degree of accuracy.

\section{METHODS OF SOLVING DIFFERENTIAL EQUATIONS}

A. General methods

$$
\begin{aligned}
& \mathrm{Y} 1(1)=\mathrm{y} 0+\frac{h}{2}[\mathrm{f}(\mathrm{x} 0, \mathrm{y} 0)+\mathrm{f}(\mathrm{x} 1, \mathrm{y} 1(0))] \\
& \mathrm{Y} 1(2)=\mathrm{y} 0+\frac{h}{2}[\mathrm{f}(\mathrm{x} 0, \mathrm{y} 0)+\mathrm{f}(\mathrm{x} 1, \mathrm{y} 1(1))] \\
& \mathrm{Y} 1(3)=\mathrm{y} 0+\frac{h}{2}[\mathrm{f}(\mathrm{x} 0, \mathrm{y} 0)+\mathrm{f}(\mathrm{x} 1, \mathrm{y} 1(2))]
\end{aligned}
$$

Each of the succeeding approximations are better than the preceding ones. They are called corrected values. Euler's formula and modified Euler's formula jointly are also called as Euler's predictor and corrector formulae.

D. Types of Euler's method

- Forward Euler's method

Integration is directly or indirectly involved in the process of getting a solution of the given D.E and accordingly the solution will be involved with arbitrary constants. Such a solution is called as the General solution of the D.E. it is obvious that the number of arbitrary constants present in the solution is equal to the order of the D.E.

- By using general methods only a particular type of differential equations can be solved. To solve complicated differential equations general methods cannot be employed.

- General methods cannot be implemented by using computers.

B. Numerical methods

We know the various analytical methods of solving differential equations, applicable only to equations in some specific form but the differential equations arising out of many physical problems do not belong to a specific form and sometimes analytical solution may not even exist. In some cases it may be very difficult to solve by analytical methods. In such cases "Numerical methods" assumes importance and computers help in many numerical methods for obtaining the result to the highest degree of accuracy.

C. Euler's method

Given a differential equation (D.E.) of the form

$$
\frac{d y}{d x}=f(x, y)
$$

and an initial condition ( $\mathrm{x} 0, \mathrm{y} 0)$, we would like to a find function $y=f(x)$ that satisfies the D.E. and passes through the Point $(\mathrm{x} 0, \mathrm{y} 0)$. In some cases, one can identify the general solution to the D.E. by inspection or using methods such as separation of variables and integration. But sometimes, either we don't have the analytic tools in our tool bag to solve the D.E. or an analytic solution is not possible. That's when we need a numerical method for solving the D.E. One such method is Euler's Method. It is based on using tangent lines to "piece together" an approximation to the particular Solution to D.E. Euler's formula is given by

$$
\mathrm{Y} 1(0)=\mathrm{y} 0+\mathrm{hf}(\mathrm{x} 0, \mathrm{y} 0)
$$

Since the accuracy is poor in this formula this value $y 1$ is successively improved to desired degree of accuracy by

- Backward Euler's method

Example:

Consider the ordinary differential equation,

$$
\frac{d x}{d t}=-X
$$

With the initial condition $\mathrm{x}(0)=1$.

Forward method is given by,

$$
\begin{gathered}
\dot{x}=\frac{x_{n+1}-x_{n}}{h} \\
\text { So, } x_{n+1}=x_{n}(1-h)
\end{gathered}
$$

Backward method is given by,

$$
\begin{gathered}
\dot{x}=\frac{x_{n}-x_{n-1}}{h} \\
\text { So, } x_{n-1}=x_{n}(\mathrm{~h}+1)
\end{gathered}
$$

E. Trapezoidal method:

The trapezoidal rule is a numerical method to solve ordinary differential equations derived from the trapezoidal rule for computing integrals. It is an implicit second-order method.

Suppose that we want to solve the differential equation

$$
y^{\prime}=f(t, y)
$$

The trapezoidal rule is given by the formula

$$
y_{(n+1)}=y_{n}+\frac{1}{2} h\left(f\left(t_{n}, y_{n}\right)+f\left(t_{n+1}, y_{n+1}\right)\right)
$$

Where, $\mathrm{h}=t_{n+1}-t_{n}$ is the step size.

The waveform of a trapezoidal rule is given by

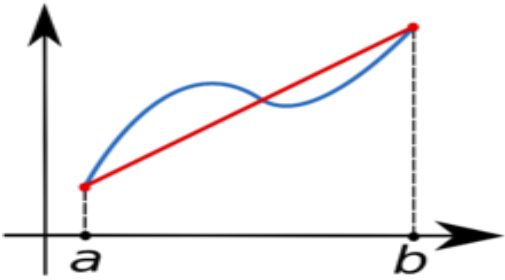

Fig 8: Trapezoidal Rule

Fourth order RK method

Consider the initial value problem $\frac{d y}{d x}=f(x, y), y\left(x_{0}\right)=$ $y_{0}$. we need find $\mathrm{y}\left(x_{0}+h\right)$ where $\mathrm{h}$ is the step size.

We have first compute $k_{1}, k_{2}, k_{3}, k_{4}$ by the following formulae.

$$
k_{1}=\mathrm{hf}\left(x_{0}, y_{0}\right)
$$


INTERNATIONAL JOURNAL OF INNOVATIVE RESEARCH IN ELECTRICAL, ELECTRONICS, INSTRUMENTATION AND CONTROL ENGINEERING Vol. 4, Issue 2, February 2016

The required,

$$
\begin{aligned}
& k_{2}=\mathrm{hf}\left(x_{0}+\frac{h}{2}, y_{0}+\frac{k_{1}}{2}\right) \\
& k_{3}=\mathrm{hf}\left(x_{0}+\frac{h}{2}, y_{0}+\frac{k_{2}}{2}\right) \\
& k_{4}=\mathrm{hf}\left(x_{0}+h, y_{0}+k_{3}\right)
\end{aligned}
$$

$$
y\left(x_{0}+h\right)=y_{0}+\frac{1}{6}\left(k_{1}+2 k_{2}+2 k_{3}+k_{4}\right)
$$

F. Realization of Low-pass filter:

A low-pass filter is a filter that passes signals with a frequency lower than a certain cutoff frequency and attenuates signals with frequencies higher than the cutoff frequency. The amount of attenuation for each frequency depends on the filter design. The filter is sometimes called a high-cut filter.

Low-pass filters exist in many different forms, including electronic circuits, anti-aliasing filters for conditioning signals prior to analog-to-digital conversion, digital filters for smoothing sets of data, acoustic barriers, blurring of images, and so on. The moving average operation used in fields such as finance is a particular kind of low-pass filter, and can be analyzed with the same signal processing techniques as are used for other low-pass filters. Low-pass filters provide a smoother form of a signal, removing the short-term fluctuations, and leaving the longer-term trend.

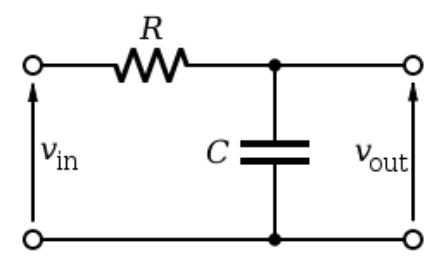

Fig 9: Low-Pass Filter

The cutoff frequency is given by,

$$
\mathrm{fc}=\frac{1}{2 \pi R C}
$$

From the circuit diagram to the right, according to Kirchhoff's Laws and the definition of capacitance:

$$
\begin{gathered}
V_{i n}(t)-R i(t) \frac{1}{C} \int_{0}^{t} i(t) d t=0 \\
V_{i n}(t)=R i(t)-\frac{1}{C} \int_{0}^{t} i(t) d t
\end{gathered}
$$

Applying laplace transform:

$$
V_{\text {in }}(s)=R i(s)+\frac{1}{C s} i(s)
$$

Voltage at capacitor is given by:

$$
V_{\text {out }}(t)=\frac{1}{C} \int_{0}^{t} i(t) d t
$$

Applying laplace transform:

$$
V_{\text {out }}(s)=\frac{1}{C s} i(s)
$$

$\mathrm{T} . \mathrm{F}=\frac{1}{(R C s+1)}$

$\mathrm{RC}=\mathrm{T}=\mathrm{RC}$ time constant

$\mathrm{T} . \mathrm{F}=\frac{\operatorname{Vout}(s)}{\operatorname{Vin}(s)}=\frac{1}{(T s+1)}$

Let input $V_{\text {in }}$ be a unit step function $\mathrm{u}(\mathrm{t})$

$\frac{v_{0}}{u}=\frac{1}{s T+1}$

$$
\begin{aligned}
& v_{0}(s T+1)=u \\
& v_{0} s T+v_{0}=u \\
& \mathrm{~T} \frac{d v_{0}(t)}{d t}+v_{0}(t)-u(t)=0 \\
& \frac{d v_{0}(t)}{d t}=\frac{u(t)-v_{0}(t)}{T}
\end{aligned}
$$

By using EBM

$$
\begin{aligned}
& \frac{d v_{0}(t)}{d t}=\frac{v_{0(n)}-v_{0(n-1)}}{h} \\
& \frac{v_{0(n)}-v_{0(n-1)}}{h}=\frac{u(t)-v_{0(n)}}{T} \\
& v_{0(n)}=v_{0(n-1)}+\left(\frac{h}{T}\right)\left(v_{i n}-v_{0(n-1)}\right.
\end{aligned}
$$

To have a cutoff frequency of $300 \mathrm{~Hz}$

$$
\begin{gathered}
R C=\frac{1}{2 \pi f}=\frac{1}{2 \pi * 1 * 300} \\
R C=0.00053 \mathrm{ohm}
\end{gathered}
$$

\section{G. BOOST CONVERTER}

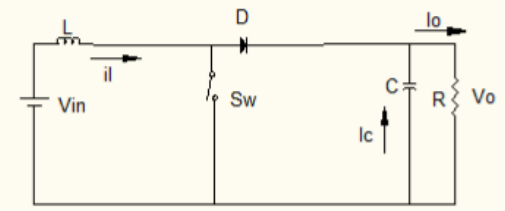

Fig 10: Boost Converter

A boost converter (step-up converter) is a DC-to-DC power converter with an output voltage greater than its input voltage. It is a class of switched-mode power supply (SMPS) containing at least two semiconductors (a diode and a transistor) and at least one energy storage element, a capacitor, inductor, or the two in combination. Filters made of capacitors (sometimes in combination with inductors) are normally added to the output of the converter to reduce output voltage ripple.

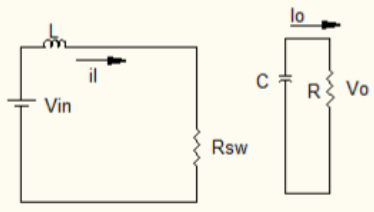

Fig 11: When Switch is closed

Applying KVL:

$\left(\mathrm{V}_{\text {in }}-\mathrm{L} \frac{\mathrm{di}}{\mathrm{dt}}=0\right) * \mathrm{dT}_{\mathrm{s}}$

Applying KCL:

$\left(\mathrm{C} \frac{\mathrm{dV}_{0}}{\mathrm{dt}}-\frac{\mathrm{V}_{0}}{\mathrm{R}_{\mathrm{l}}}=0\right) * \mathrm{dT}_{\mathrm{s}}$

When switch is open:

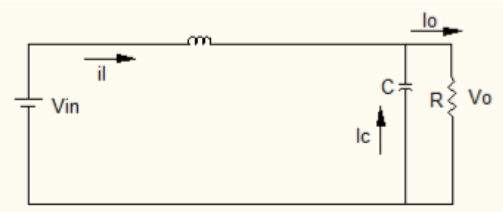

Fig 12: When switch is open 
INTERNATIONAL JOURNAL OF INNOVATIVE RESEARCH IN ELECTRICAL, ELECTRONICS, INSTRUMENTATION AND CONTROL ENGINEERING Vol. 4, Issue 2, February 2016

Applying KVL:

$$
\left(V_{\text {in }}-L \frac{d I_{1}}{d t}-V_{0}=0\right) *(1-d) T_{s}
$$

Applying KCL:

$$
\begin{aligned}
\left(\mathrm{I}_{\mathrm{l}}-\mathrm{c} \frac{\mathrm{dV_{0 }}}{\mathrm{dt}}-\frac{\mathrm{V}_{0}}{\mathrm{R}_{\mathrm{l}}}=0\right) & *(1-\mathrm{d}) \mathrm{T}_{\mathrm{s}} \\
\frac{\mathrm{dI}_{\mathrm{l}}}{\mathrm{dt}} & =\frac{(\mathrm{d}-1) \mathrm{V}_{0}+\mathrm{V}_{\text {in }}}{\mathrm{L}} \\
\frac{\mathrm{dV_{0 }}}{\mathrm{dt}} & =\frac{\mathrm{I}_{\mathrm{l}}(1-\mathrm{d})-\frac{\mathrm{V}_{0}}{\mathrm{R}_{\mathrm{l}}}}{\mathrm{C}}
\end{aligned}
$$

\section{By using EBM}

$$
\begin{gathered}
I_{l(n)}-I_{l(n-1)}=\frac{h}{L}\left[(d-1) V_{0}+V_{\text {in }}\right] \\
V_{0(n)}=V_{0(n-1)}+\frac{h}{c}\left[I_{l(n-1)}(1-d)-\frac{V_{0(n-1)}}{R_{l}}\right] \\
I_{0}=\frac{V_{0}}{R} ; I_{\text {in }}=I 1 ; I_{c}=I 1-I 0
\end{gathered}
$$

\section{RESULT}

Basic operations on signals:

5.1.1 Addition:

$\mathrm{y}(\mathrm{t})=\mathrm{x} 1(\mathrm{t})+\mathrm{x} 2(\mathrm{t})$

$\mathrm{x} 1(\mathrm{t})=5 \operatorname{sint}($ channel 1$)$

$\mathrm{x} 2(\mathrm{t})=5 \mathrm{v}$ dc signal $($ channel 2$)$
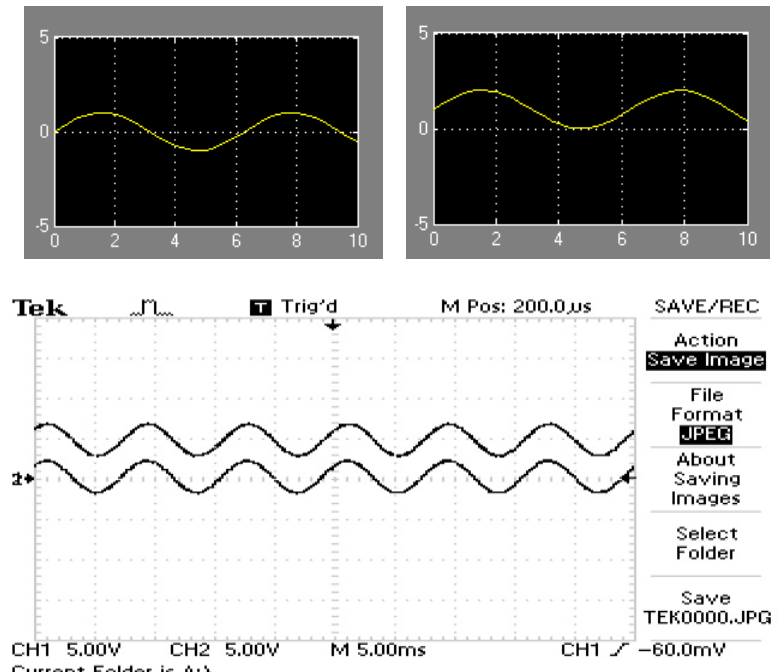

Multiplication:

$\mathrm{y}(\mathrm{t})=\mathrm{x} 1(\mathrm{t}) \cdot \mathrm{x} 2(\mathrm{t})($ channel 1$)$

$\mathrm{x} 1(\mathrm{t})=5$ sint $($ channel 2$)$

$\mathrm{x} 2(\mathrm{t})=0.5 \mathrm{v}$ de signal

MAT LAB output:
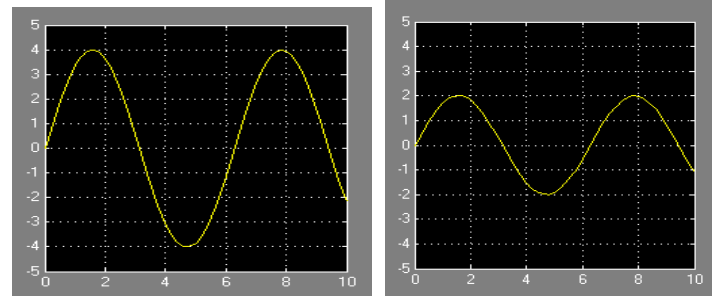

DSC chip output:

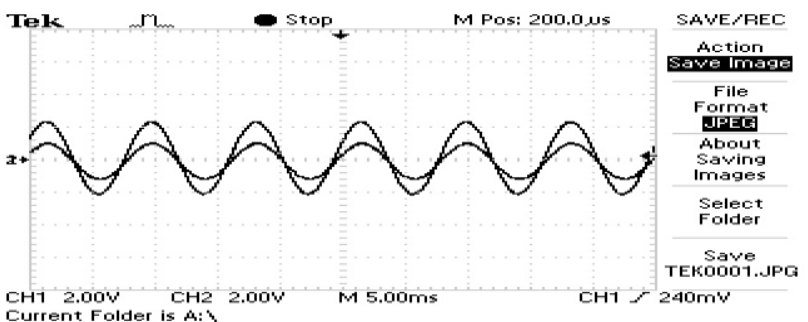

Solving differential equations:

$$
\frac{\mathrm{dx}}{\mathrm{dt}}=-\mathrm{x}, \mathrm{h}=0.01 ; \mathrm{x}_{0}=10 ;
$$

MAT LAB output:

By using,

- RK method,

- EBM,

- EFM,

- Trapezoidal method
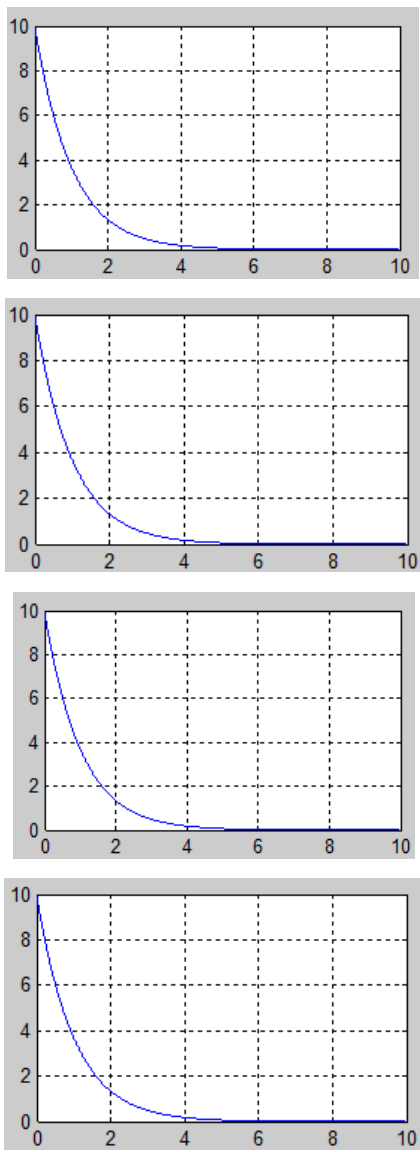

DSC chip output:

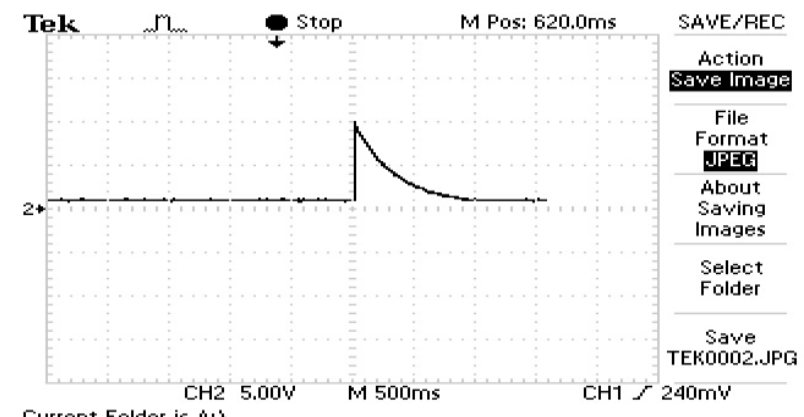

Current Folder is A: 


\section{CONCLUSION}

In this paper, a real time simulator is designed and built using DSC chip. Simulation of boost converter by deriving ideal and exact mathematical model is done. By using real time simulator it is possible to simulate power converter circuits accurately. They can be efficiently used in various fields such as industries, education, etc. With this, simulator can be used for performance optimization and safety engineering in various fields. Building a real time simulator Results obtained from real time simulation are compared with offline simulation. To design and build a real time simulator and simulate ideal and exact models of boost converter. Also accomplish tasks like performing basic operations on signals, solving differential equations, realizing low pass filter by using MAT LAB and simulator.

\section{REFERENCES}

11] Hui Zhang, Li Ming Shi, Yang Li, Ke Wang, "Real Time Simulation of High Power Converter," Page(s): 306 - 310; Print ISBN: 978-1-4244-7720-3, 10-13,Oct,2010.

[2] Hoang Le-Huy, Gilbert Sybille, Richard Gagnon, Van Que Do, "Real-Time Simulation of PWM Power Converters in a DoublyFed Induction" 1-4244-0136-4/06/2006 IEEE.

[3] R. Pena, J.C. Clare, G.M. Asher, "Doubly-fed induction generator using back-to-back PWM converters and itsapplications to variablespeed wind-energy generation," IEE Proceedings on Electrical Power Applications, Vol. 143, No. 3, May 1996, pp. 231-341.

[4] R. Gagnon et al, "Modelling and real-time simulation of a doublyfed induction generator driven by a wind turbine," in Proceedings of the International Conference on Power Systems Transients IPST'05, Montreal, June 2005, paper no. IPST05-162.

[5] J. Clerk Maxwell, A Treatise on Electricity and Magnetism, 3rd ed., vol. 2. Oxford: Clarendon, 1892, pp. 68-73. P. Lok-Fu, et al., “A versatile cluster-based real-time digital simulator for power engineering research," Power Systems, IEEE Transactions on, Vol. 21, pp. 455-465, 2006.

\section{BIOGRAPHIES}

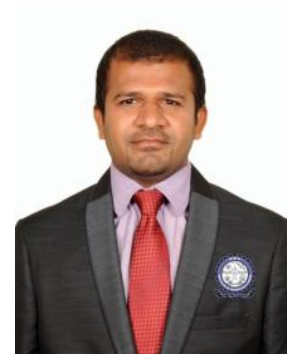

Babu Naik $\mathbf{G}$ has received his masters from the Indian Institute of Science (IISc), Bangalore, India. Currently working as Assistant professor in the department of Electrical and Electronics Engg at BMSIT\&M, Bangalore. His research interests are in the field of power systems, Power Electronics and Industrial drives and applications.

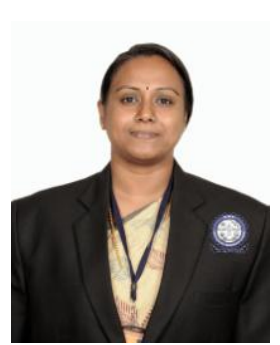

Shilpa G has received her master's from MS Ramaiah Institute Of Technology, Bangalore, India. Currently working as Assistant professor in the department of Electrical and Electronics Engg at BMSIT\&M, Bangalore. Her research interests are in the field of Power Electronics and electric drives.

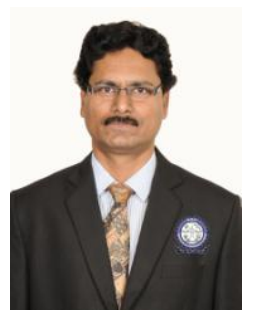

H.D Kattimani has received his masters from BITS, Pilani, Rajasthan, India. Currently working as Associate professor and HOD in the department of Electrical and Electronics Engg at BMSIT\&M Bangalore. His research interests are in the field of power systems and Electrical Machines.

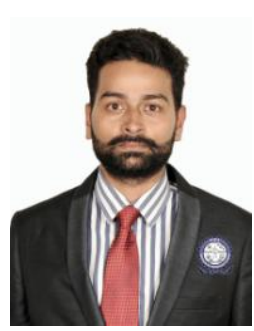

Ozwin Dominic Dsouza has received his masters from National Institute of Engineering, Mysore, India. Currently working as Assistant professor in the department of Electrical and Electronics Engg at BMSIT\&M, Bangalore. His research interests are in the field of electric drives and control systems. 